\title{
Isolated sedoheptulokinase deficiency
}

\author{
INSERM
}

\section{Source}

INSERM. (1999). Orphanet: an online rare disease and orphan drug data base. Isolated sedoheptulokinase deficiency. ORPHA:440713

A rare, hereditary disorder of pentose phosphate metabolism characterized by increased urine levels of sedoheptulose and erythtirol, and low-to-normal excretion of sedoheptulose-7P. Clinical presentation of this disorder is currently unclear. 